\title{
AUTISMO INFANTIL:ACOLHIMENTO E TRATAMENTO PELO SISTEMA ÚNICO DE SAÚDE
}

\section{CHILDHOOD AUTISM: RECEPTION AND TREATMENT BYTHE UNIFIED HEALTH SYSTEM}

\author{
Mayara Andrine do Nascimento* \\ mayaraan@gmail.com \\ Marília Pereira* \\ mariliaampereira@gmail.com \\ Sônia Cardoso Moreira Garcia* \\ sph1918@hotmail.com \\ * Centro Universitário de Volta Redonda/RJ, Brasil
}

\begin{abstract}
Resumo
O presente artigo trata de revisão de literatura e versa sobre o diagnóstico do Transtorno do Espectro Autista em crianças, o acolhimento oferecido à família e ao tratamento multidisciplinar oferecidos pelo SUS - Sistema Único de Saúde, em âmbito nacional. O referido tema torna-se objeto deste estudo, pois não há publicações que correlacionem os uni termos de maneira integrativa às várias áreas profissionais nos bancos de dados SCIELO, MEDLINE, PUBMED e LILACS, utilizando palavras em português, inglês ou espanhol, no período de 10 anos. Para isso, a pesquisa será norteada pelas Dimensões Novikoff (2010). O autismo caracteriza-se pelo desenvolvimento atípico e inadequado das linguagens e comunicação, meio social e emocional, além de apresentar interesses e atividades restritas. Devido ao crescente aumento do diagnóstico de transtornos neuropsiquiátricos, torna-se necessário que profissionais em todas as áreas da saúde atuem em equipe, para um completo amparo à criança $\mathrm{e}$ sua família. O acolhimento global deve ser assegurado, a todas as famílias, pelo SUS e, devido à escassez de artigos multidisciplinares na área, este estudo torna-se relevante uma vez que se propõe compreender se há, de fato, um amparo oferecido aos portadores de autismo.
\end{abstract}

Palavras-chave: Autismo infantil. Acolhimento. SUS.

\begin{abstract}
This article deals with a literature review and deals with the diagnosis of Autistic Spectrum Disorder in children, the reception offered to the family and the multidisciplinary treatment offered by SUS - National Health System, at a national level. This subject becomes the object of this study, since there are no publications that correlate the uni terms in an integrative way to the various professional areas in the databases SCIELO, MEDLINE, PUBMED and LILACS, using words in Portuguese, English or Spanish, in the period of 10 years. For this, the research will be guided by the Dimensions Novikoff (2010). Autism is characterized by the atypical and inappropriate development of languages and communication, social and emotional environment, as well as presenting restricted interests and activities. Due to the growing increase in the diagnosis of neuropsychiatric disorders, it is necessary for professionals in all areas of health to work as a team, for a complete support to the child and his family. The global reception should be assured to all families by the SUS and, due to the scarcity of multidisciplinary articles in the area, this study becomes relevant since it proposes to understand if there is, in fact, an support offered to the autism carriers .
\end{abstract}

Keywords: Chieldhood Autism. Reception. Public Health Service. 


\section{INTRODUÇÃO}

O presente artigo trata de revisão de literatura e versa sobre o diagnóstico do Transtorno do Espectro Autista em crianças, o acolhimento oferecido à família e ao tratamento multidisciplinar oferecidos pelo SUS - Sistema Único de Saúde, em âmbito nacional. São objetos deste estudo, pois são escassas publicações que correlacionem ambos os temas de maneira integrativa às várias áreas profissionais nos bancos de dados SCIELO, MEDLINE, PUBMED e LILACS, utilizando palavras em português, inglês ou espanhol, no período de 20 anos.

$\mathrm{O}$ autismo caracteriza-se como um transtorno neuropsiquiátrico que se desenvolve na infância precoce (AMARAL,PORTILLO\& MENDES, 2011) e, na maioria das vezes, até os três anos de idade (ZAMPIROLI \& SOUZA, 2012). Ele se insere como um dos Transtornos Globais do Desenvolvimento que incluem, além do Transtorno do Espectro Autista, a Síndrome de Rett, o Transtorno de Asperger, o Transtorno Desintegrativo da Infância e Transtorno Global do Desenvolvimento sem outra especificação (FURTADO,BRAYNER\&SILVA, 2014).

O Transtorno do Espectro Autista caracteriza-se pelo desenvolvimento atípico e inadequado das linguagens e comunicação - devido à demora na aquisição da linguagem e uso repetitivo da fala -, meio social - no qual apresenta pouca reciprocidade ao que lhe apresentam - e emocional, além de apresentar interesses e atividades restritas (CAMARGO\& BOSA, 2009; MARCELLI, 1998 apud SMEHA\& CEZAR, 2009). Vale relatar que, dentro do Autismo Infantil, há dois subtipos: de auto e de baixo rendimento ou funcionamento, sendo o de baixo rendimento o subtipo clássico, consequentemente, o mais frequente, conhecido e estudado (CAMARGOS JUNIOR, 2010). Tais características comportamentais dificultam a relação do portador do autismo com as pessoas que o cercam sejam estas familiares ou profissionais de saúde, o que pode tornar difíceis e até desinteressantes, o tratamento e acompanhamento das crianças portadoras dessa condição.

Nas últimas décadas, especialmente nos últimos vinte anos vêm ocorrendo aumento expressivo dos diagnósticos de transtornos de origem neurobiológica na infância. Especialmente na última década, subiu consideravelmente o número de casos de crianças portadoras de Transtornos Globais do Desenvolvimento, e dentre eles, o Transtorno do Espectro Autista (UNTOIGLICH, 2013). Santos et al (2005) relatam diversos estudos que demonstram que tais transtornos variam e ainda, relatam uma prevalência de 10 a $12 \%$ de crianças, em grupos de 5 ou 7 a 14 anos, com problemas mentais (SANTOS et al, 2005). Zampiroli e Souza (2012), por outro lado, apontam para estudos que relatam a incidência do Autismo Infantil entre 2 a 5 casos a cada 10.000 crianças, tendo alguns citados, como Lord et al (1997) defendido a proporção de 1 caso a cada 500 crianças em um de seus estudos 
(LORD et al, 1997 apud ZAMPIROLI \& SOUZA, 2012). Torna-se evidente, a partir dessas estatísticas, portanto, que tanto profissionais como Sistemas de Saúde devem estar preparados para receber, acolher e oferecer os tratamentos e serviços adequados para os indivíduos autistas e sua rede familiar.

O atual Sistema Único de Saúde do Brasil - SUS, que foi criado em 1988 pela Constituição Federal promulgada no mesmo ano - a chamada "Constituição Cidadã" -, tem por princípio oferecer este acolhimento e tratamento necessários, assegurados pelo artigo 196, que ressalta ser, a saúde, direito de todos e dever do Estado. Complementar a isso, conforme o artigo 7 da lei 8.080/90, o Sistema baseia-se em três pilares principais, quais sejam: a Universalidade, que assegura a saúde como direito de todos os cidadãos e o acesso aos serviços e ações de saúde indiscriminado a todos; a Equidade, na qual todos os cidadãos são iguais perante o SUS que, entretanto, atende cada um conforme suas necessidades particulares e individuais - mais assistência aos que demandam mais necessidades em saúde; e a Integralidade, ou seja, a capacidade que o Sistema dever ter de oferecer atenção à todas as demandas da população, independente de quão complexas sejam (BRASIL. Lei 8.080, 1990, art. 7; SILVA \& SENNA, 2013). Para dinamizar o processo de atendimento, o SUS foi hierarquizado em setores primário, secundário e terciário, de acordo com os níveis de complexidade, onde o setor primário corresponde à porta de entrada para o Sistema - Unidades Básicas de Saúde, o setor secundário inclui serviços médicos especializados, de apoio diagnóstico e terapêutico e atendimento de urgência e emergência, e o setor terciário inclui procedimento de alta tecnologia e alto custo (MINISTÉRIO DA SAÚDE, 2009; ERDMANN et al, 2013).

A organização do Sistema se dá por meio de áreas geográficas regionalizadas, na qual atuam as Equipes Multiprofissionais de Saúde da Família, que devem se preocupar em promover saúde, através de atividades diversas que levem em conta a diversidade cultural e social de cada região. A promoção da saúde pode ser compreendida como um processo no qual o indivíduo alcança o completo bem-estar físico, mental e social a partir da identificação de aspirações, satisfação de necessidades e interação com o ambiente. Dessa forma, a saúde pode ser vista como um meio de vida e não um objetivo (MINISTÉRIO DA SAÚDE, 1986 apud AMARAL; PORTILLO \& MENDES, 2011). Além disso, deve-se ter em mente que, além das necessidades próprias de sua condição, pessoas com deficiência têm necessidades básicas comuns, como, por exemplo, vacinação, consultas, pré-natal, puericultura e saúde bucal (MINISTÉRIO DA SAÚDE, 2009 apud AMARAL; PORTILLO \& MENDES, 2011).

Além dessas referidas acima, indivíduos portadores do Transtorno do Espectro Autista apresentam necessidades em saúde específicas de sua condição. Nesse sentido, existem os CAPS - Centro de 
Atenção Psicossocial -, nos quais profissionais especializados promovem ações em saúde e tratamento para portadores dos mais diversos transtornos psicossociais. Eles funcionam dentro do contexto da Política Nacional de Saúde Mental e se apoia na Lei 10.216/01, cujo principal objetivo garante um serviço de base à Saúde Mental de alicerce comunitário, permitindo que os indivíduos com transtornos mentais circulem livremente pelos serviços, comunidade e cidade (BRASIL. Lei 10.216, 2001). Uma das modalidades do CAPS, o CAPSi - Centro de Atenção Psicossocial Infantil -, cuja atenção volta-se à infância e adolescência e auxilia, por exemplo, no acompanhamento do Autismo Infantil, tem se expandido. Sua forma de organização estimula a integração social e familiar e ajuda crianças e adolescentes a estabelecerem suas autonomias. (MINISTÉRIO DA SAÚDE, 2007 apud EBERT, LORENZINI E SILVA, 2015).

Dessa forma, tanto a criança portadora do Transtorno do Espectro Autista quanto sua família, merecem e têm direito à atenção e acolhimento integral pelo SUS, uma vez que são cidadãos com os mesmos direitos assegurados por Lei. Em consonância com essa necessidade de acolhimento, em 2012 foi instituída uma Política Nacional de Proteção dos Direitos da Pessoa com Transtorno do Espectro Autista, visando garantir a Integralidade na assistência à saúde aos indivíduos portadores de tal condição. (FURTADO, BRAYNER \& SILVA, 2014). O Sistema e Equipe de Saúde devem, em suas ações, integrar promoção de saúde, atendendo às demandas básicas desses pacientes, com o cuidado acolhedor específico que o Transtorno requer.

Por fim, entende-se que o diagnóstico do autismo infantil, o acolhimento da criança e de sua família, de maneira global, e seu tratamento devem ser assegurados pelo Sistema Único de Saúde. O presente artigo foi elaborado devido a escassa produção de material que discurse e dialogue de maneira multidisciplinar sobre os direitos citados, suscitando as questões: este acolhimento existe e mostra-se eficiente no amparo às crianças autistas e suas famílias? Há uma relação multiprofissional horizontal, com comunicação entre profissionais de maneira clara e direta, visando complementar o tratamento do autista? Os profissionais estão realmente capacitados para que esta logística funcione adequadamente, de forma a não prejudicar o paciente? Com isto, entende-se que a função deste artigo será integrar as produções, sugerindo a organização de produto de acolhimento eficaz a ser implementado, caso o atual modelo mostre-se ineficiente.

\section{OBJETIVOS}

\subsection{Objetivo Geral}


Compreender o funcionamento do Sistema Único de Saúde Brasileiro nem relação ao acolhimento e trato de crianças com Transtorno do Espectro Autista e suas famílias, em âmbito nacional.

\subsection{Objetivos Específicos}

Para alcançar a avaliação desejada do funcionamento do SUS, torna-se necessário também:

- conhecer a postura dos profissionais em saúde no atendimento de crianças autistas e seus familiares;

- identificar a eficiência e funcionalidade tanto das Unidades Básicas em Saúde, que são a porta de entrada do Sistema, quanto dos CAPSi, e o processo de referenciamento das crianças de uma unidade para outra;

- apontar a eficiência do trabalho multiprofissional no atendimento das referidas crianças, tanto no mesmo nível de atenção quanto entre os níveis;

- reconhecer o acompanhamento e suporte dado à família em todo esse processo;

\section{JUSTIFICATIVA}

Reconhecer que existe uma parcela significativa de crianças portadoras do Transtorno do Espectro Autista, que têm direito ao acompanhamento adequado em saúde, torna-se de extrema importância. Nesse sentido, o presente trabalho auxilia na identificação de possíveis equívocos nesse processo, indicando caminhos possíveis para um atendimento mais holístico e humanizado, bem como integral.

A produção e conhecimento em Saúde ainda são feitos de forma muito fragmentada, evidenciandose a falta de comprometimento com o cuidado integral do indivíduo. O autismo, na maioria das produções acadêmicas está relacionado com uma área específica do cuidado - Pediatria, Fonoaudiologia, Psicologia, Odontologia, entre outros - deixando em segundo plano o acompanhamento integral que tal condição requer (FURTADO, BRAYNER \& SILVA, 2014).

Além disso, ainda se produz pouco sobre a referência e relacionamento do fenômeno dos Transtornos Globais de Desenvolvimento com a Saúde Pública (FURTADO, BRAYNER \& SILVA, 2014). O artigo em tela pode contribuir com os relatos a respeito e ajudar a elucidar o processo diagnóstico-acolhimento-acompanhamento da criança autista referenciada ao SUS, salientando a importância da integração multiprofissional.

Tal integração se mostra necessária e também demonstra a contribuição que o presente artigo pode trazer, entre outros, para profissionais e estudantes da área de saúde. Muitos destes profissionais 
trabalham no Sistema Único de Saúde - maior empregador em saúde no Brasil para diversas profissões (SILVA \& SENNA, 2013) - e devem mostrar-se preparados para acolher e tratar todos os pacientes, como as crianças portadoras de autismo.

E ainda, a população em geral, a partir dessa leitura, pode pensar e agir de forma mais consciente acerca dos seus direitos. Entender a lógica e funcionamento do Sistema se torna fundamental para que a tal instância, a população e o usuário, se façam presentes no processo de construção, aprimoramento e efetivação do Sistema Único de Saúde, uma vez que a Participação Comunitária é parte das Diretrizes preconizadas do SUS (SILVA \& SENNA, 2013).

\section{METODOLOGIA}

O presente artigo trata-se de uma revisão sistemática da literatura, uma vez que busca, através da base literária, responder criticamente se existe e se o eixo criança portadora de autismo-família recebe o apoio necessário pelo Sistema único de Saúde (SAMPAIO \& MANCINI, 2007). Sua construção ocorreu devido a não serem encontrados artigos com os mesmo objetivos que este.

Para isso, no período de fevereiro de 2015 a outubro de 2016, foram selecionados artigos disponíveis nas plataformas SCIELO, MEDLINE, PUBMED e LILACS que relacionassem autismo infantil e SUS, além de serem utilizadas as cartilhas e orientações do Ministério da Saúde brasileiro, disponíveis em suas plataformas online. O estudo foi feito da seguinte forma: foram realizadas diversas pesquisas nas bases de dados cruzando diferentes termos em português, tais como "Autismo Infantil" e "SUS"; “"Autismo Infantil", "SUS” e "Brasil”; "Autismo Infantil" e "Brasil"; “Autismo Infantil” e "Serviço Público"; “Autismo Infantil” e "Saúde Pública”, e termos em inglês, na plataforma PUBMED como "Chieldhood Autism" e "Public Service" e "Childhood Autism" e "Public Health".

Dos trabalhos encontrados e disponíveis para leitura gratuita, foram lidos seus resumos, sendo selecionados para discussão os artigos que apresentam em seu conteúdo uma associação direta entre os termos pesquisados, tornando-se relevantes para a revisão aqui apresentada.

Foram excluídos os artigos que, embora contemplassem o tema Autismo Infantil, não abordassem uma visão pela saúde pública e nem apresentassem uma visão multidisciplinar sobre o acolhimento da criança e sua família.

Da mesma forma, buscou-se por leis referentes aos direitos do cidadão e deveres do SUS, assim como também foram utilizadas cartilhas publicadas pelo Ministério da Saúde, que abordassem o tema do Autismo Infantil. O presente estudo terá como desenho de pesquisa as "Dimensões propostas por Novikoff (2010)", um estudo da Professora Cristina Novikoff voltado à pesquisas de 
ensino superior, a fim de superar manuais e propor um novo processo de planejamento, realização e textualização de forma não linear, mas didático.

\section{RESULTADOS E DISCUSSÃO}

Considerando os métodos empregados para a pesquisa, obtiveram-se poucos artigos de importância para este estudo, o que representa um espaço de conhecimento e de informações, escasso.

Na plataforma Scielo, ao cruzar "Autismo Infantil" e "Serviço Público", da mesma forma, não houve evidências de trabalhos realizados. Porém, ao cruzar as palavras "Autismo Infantil" e "SUS", foram encontrados dez artigos os quais não evidenciaram afinidades com a discussão aqui proposta. Ao cruzar "Autismo Infantil" e "Saúde Pública", apenas um artigo foi encontrado, porém também sem evidências de diálogo com o objeto de estudo aqui elencado.

Ao pesquisar no banco de dados da MEDLINE, cruzou-se as palavras "Autismo Infantil" e "Brasil", assim como as palavras "Autismo Infantil", "SUS" e "Brasil; "Autismo Infantil" e "Serviço Público"; e "Autismo Infantil" e "Saúde Pública", não obtendo resultados.

A pesquisa na plataforma LILACS obteve alguns resultados satisfatórios. Ao pesquisar as palavras "Autismo Infantil", "SUS" e "Brasil", foi encontrado apenas um artigo de grande relevância para o presente estudo, publicado pela Physis, revista de saúde coletiva, em 2014. Ao pesquisar apenas as palavras "Autismo Infantil" e "Brasill", foram obtidos vinte e três artigos, sendo destes, dois de relevância: uma tese de doutorado publicada pela USP, em 2014, e o mesmo artigo citado anteriormente, encontrado com a pesquisa das mesmas palavras, acrescidas do vocábulo "SUS". Ao cruzar as palavras "Autismo Infantil" e "Saúde Pública", foi encontrado um artigo de interesse, porém com acesso apenas ao resumo, por conta disso, este artigo foi excluído da pesquisa. Por fim, pesquisaram-se as palavras "Autismo Infantil” e "Serviço Público", unitermos estes que não trouxeram achados.

Por último, a plataforma PUBMED encontrou cento e dezessete artigos ao cruzar as palavras "Chieldhood Autism" e "Brazil”, porém nenhum que possuísse relação com o SUS. Ao cruzar estas palavras com os vocábulos "SUS" ou "Sistema Único de Saúde", nenhum artigo foi encontrado. Foi pesquisado também as palavras "Chieldhood Autism" e "Public Service", encontrando um artigo sem relevância, e "Chieldhood Autism" e "Public Health", que apontou doze artigos, também sem relevância. 
QUADRO 1: Artigos de relevância para o artigo encontrados pelas pesquisas nas plataformas.

\begin{tabular}{|l|l|}
\hline Autor, ano de publicação, cidade & Título \\
\hline Lima, 2014, Rio de Janeiro & $\begin{array}{l}\text { Indicadores sobre o cuidado a crianças e } \\
\text { adolescentes com autismo na rede de CAPSi } \\
\text { da região metropolitana do Rio de Janeiro }\end{array}$ \\
\hline Netrval, 2014, São Paulo & $\begin{array}{l}\text { Proposta de modelo de indicadores de } \\
\text { qualidade para o atendimento oferecido aos } \\
\text { indivíduos autistas na cidade de São Paulo }\end{array}$ \\
\hline
\end{tabular}

\subsection{Histórico do Acolhimento e Tratamento da Criança Autista}

Para uma melhor compreensão sobre o tema e sobre a importância evidente do presente estudo, relata-se um breve histórico do acolhimento e tratamento da criança diagnosticada com Transtorno do Espectro Autista, tomando como referência um dos artigos relevantes encontrados, a Lei $\mathrm{n}^{\circ}$ 12.764/2012 e as diretrizes de atenção à reabilitação da pessoa com Transtorno do Espectro Autista (TEA), publicada pelo Ministério da Saúde.

Crianças autistas eram, até o final o século passado, mantidas fora da área da saúde mental, sendo cuidadas pelas próprias escolas, assistentes sociais, entidades filantrópicas ou serviços montados por associações familiares. No sistema público de saúde, essas crianças eram atendidas por "serviços-ilha" ou como pacientes tradicionais nos ambulatórios, sendo o principal tratamento por via medicamentosa. Devido ao pequeno interesse, a maioria dos pacientes portadores do Transtorno do Espectro Autista mantinham-se sem cuidados (LIMA, 2014).

Entre 1990 e 2000, uma instituição de Betim-MG, a CERSAMI, inspirou a proposição dos CAPSi, que é um serviço multidisciplinar com o objetivo de oferecer atenção diária e intensiva a jovens com graves transtornos mentais. Embora o CAPSi não seja uma instituição exclusiva ao autismo, pode-se dizer que este foi o primeiro passo na inclusão do Transtorno do Espectro Autista ao campo da saúde mental no Brasil. A partir deste momento, o autismo começou a ser visto como um real problema de desenvolvimento psicossocial e leis e diretrizes passaram a ser elaborados, tais como a Lei $\mathrm{n}^{\mathrm{o}} 12.764 / 2012$, que institui a política nacional de proteção dos direitos da pessoa com 
Transtorno do Espectro Autista, e documentos do Ministério de Saúde sobre o atendimento a pessoas com autismo no SUS (LIMA, 2014; BRASIL. Lei 12.764, 2012).

O Ministério da Saúde publicou, em 2013, diretrizes de atenção à reabilitação da pessoa com Transtorno do Espectro Autista, onde reforça que, para atenção integral ao paciente, todas as ações elucidadas na cartilha devem ser articuladas com outros pontos de atenção da rede do SUS (atenção básica, especializada e hospitalar), bem como os serviços de proteção social (centros dia, residências inclusivas, CRAS e CREAS), e de educação (MINISTÉRIO DA SAÚDE, 2013).

\section{CONCLUSÕES}

Ao analisar os textos encontrados pelas pesquisas, percebe-se que pouco se discorre sobre como deve ser o acolhimento e tratamento oferecidos aos pacientes com Transtorno do Espectro Autista, de forma multidisciplinar, devido a sua recente inclusão ao campo de doenças mentais. Muitos estudos apresentam o tema, mas relacionados a apenas uma das áreas de interesse, como medicina, psicologia, odontologia ou fonoaudiologia.

Atualmente, a criança atendida em ambulatórios que recebe o diagnóstico do autismo é imediatamente referenciada ao CAPSi, onde irá receber o amparo necessário, dispondo de psiquiatras, fonoaudiólogos, enfermeiros, psicólogos, terapeutas e assistentes sociais. Embora este acolhimento ainda não seja o suficiente para atender de forma global todas as necessidades da criança autista, é perceptível que houve um grande avanço devido ao pouco tempo em que esta deficiência foi aceita como doença mental.

Faltam ainda, para os pacientes em tratamento, uma estrutura capaz de abranger de forma eficaz e integrativa a educação dessas crianças, que ainda são submetidas ao ensino tradicional nas escolas, e que muitas das vezes não possuem profissionais qualificados para acompanhamento do seu desenvolvimento. Também é necessário implementar o apoio oferecido à família da criança com Transtorno do Espectro Autista, que têm suas rotinas alteradas devido à doença, diminuindo carga horária de trabalho e lazer.

Por fim, salienta-se a importância da pesquisa sobre o acolhimento e tratamento dos pacientes diagnosticados com autismo, de forma multidisciplinar, para que possam ser localizados os aspectos de fragilidade do sistema, apontando para as autoridades competentes fatores a serem aprimorados, para que estas ofereçam um melhor serviço de amparo não só ao paciente, mas também a sua família. O referido estudo não se esgota em si mesmo mas vem provocar e instigar reflexões acerca do tema proposto e assim, na articulação entre a teoria e a prática, viabilizar efetivas ações as quais possam dar conta do proposto. 
Nascimento et al. 


\section{REFERÊNCIAS}

AMARAL, Lais David; PORTILHO, Jorge Alberto Cordón; MENDES, Silvia Carolina Teixeira. Estratégias de acolhimento e condicionamento do paciente autista na Saúde Bucal Coletiva. Tempus Actas de Saúde Coletiva, [S.1.], v. 5, n. 3, p. pg. 105-114, dez. 2011. ISSN 19828829. Disponível em <http://tempusactas.unb.br/index.php/tempus/article/view/1046>. Acesso em 28 de Abril de 2016.

BRASIL. Constituição (1988). Constituição da República Federativa do Brasil: promulgada em 5 de outubro de 1988. Art. 196. Disponível em <http://www.jusbrasil.com.br/topicos/920107/artigo196-da-constituicao-federal-de-1988> Acesso em 03 de Maio de 2016.

BRASIL. MINISTÉRIO DA SAÚDE. Lei n. ${ }^{\circ}$ 10216, de 06 de abril de 2001. Dispõe sobre a proteção e os direitos das pessoas portadoras de transtornos mentais e redireciona o modelo assistencial em saúde mental. Lex-Legislação em Saúde Mental 1990-2004, Brasília, 5.ed. amp., p. 17-19, 2004.

Lei $\mathrm{n}^{\circ}$ 8080, de 19 de setembro de 1990. Dispõe sobre as condições para a promoção, proteção e recuperação da saúde, a organização e o funcionamento dos serviços correspondentes e dá outras providências. Diário Oficial da União, Brasília, DF, 20 de setembro de 1990.

Lei n ${ }^{\circ}$ 12.764, de 27 de dezembro de 2012. Institui a Política Nacional de Proteção dos Direitos da Pessoa com Transtorno do Espectro Autista; e altera o $\S 3^{\circ}$ do art. 98 da Lei no 8.112, de 11 de dezembro de 1990. Diário Oficial da União, Brasília, DF, 28 de dezembro de 2012.

CAMARGO, Síglia Pimentel Höher; BOSA, Cleonice Alves. Competência social, inclusão escolar e autismo: revisão crítica da literatura. Psicol. Soc., Florianópolis , v. 21, n. 1, p. 65-74, Apr. 2009 . Disponível em <http://www.scielo.br/scielo.php?script=sci_arttext\&pid=S0102$71822009000100008 \& \operatorname{lng}=$ en\&nrm=iso> . Acesso em 28 de Abril de 2016.

CAMARGOS JUNIOR, Walter. Custo familiar com autismo infantil. Belo Horizonte.Instituto de Previdência dos Servidores de Estado de Minas Gerais, 2010. Disponível em <http://www.psiquiatriainfantil.com.br/teses/WalterCamargosJunior.pdf $>$ Acesso em 28 de Abril de 2016.

EBERT, Michele; LORENZINI, Elisiane; SILVA, Eveline Franco da. Mothers of children with autistic disorder: perceptions and trajectories. Rev. Gaúcha Enferm., Porto Alegre, v. 36, n. 1, p. 49-55, Mar. 2015. Disponível em <http://www.scielo.br/scielo.php?script=sci_arttext\&pid=S1983-

14472015000100049\&lng=en\&nrm=iso>. Acesso em 28 de Abril de 2016.

ERDMANN, Alacoque Lorenzini; ANDRADE, Selma Regina de; MELLO, Ana Lúcia Schaefer Ferreira de; DRAGO, Lívia Crespo. A atenção secundária em saúde: melhores práticas na rede de serviços. Rev. Latino Americana de Enfermagem. Ribeirão Preto. v. 21, n. spe, p. 131-139, Fev.2013. Disponível em <http://www.scielo.br/scielo.php?script=sci_arttext\&pid=S0104-

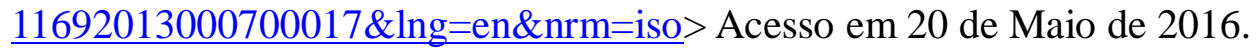


FURTADO, Luis Achilles; BRAYNER, José Clauber; SILVA, Lucas Silveira. Transtornos globais de desenvolvimento e saúde pública: uma revisão integrativa. Rev. Percurso Acadêmico. Belo Horizonte. v. 4, n. 8, p. 283-297, jul/dez. 2014. Disponível em <http://200.229.32.55/index.php/percursoacademico/article/view/8000/8684> Acesso em $28 \mathrm{de}$ Abril de 2016.

LIMA, Rossano Cabral et al . Indicadores sobre o cuidado a crianças e adolescentes com autismo na rede de CAPSi da região metropolitana do Rio de Janeiro. Physis, , v. 24, n. 3, p. 715-739, set. 2014. Disponível em <http://www.scielosp.org/scielo.php?script=sci_arttext\&pid=S0103$73312014000300715 \& \operatorname{lng}=$ pt\&nrm=iso > Acesso em 04 de Outubro de 2016.

MINISTÉRIO DA SAÚDE. Portal da Saúde (Brasília, DF). Mais sobre serviços disponíveis em saúde mental. Disponível em <http://portalsaude.saude.gov.br/index.php/cidadao/acoes-eprogramas/conte-com-a-gente/leia-mais-conte-com-a-agente> Acesso em 15 de Maio de 2016.

MINISTÉRIO DA SAÚDE. Conselho Nacional de Secretarias Municipais de Saúde (Brasília,DF). O SUS de A a Z: Garantindo saúde nos municípios. ISBN 978-85-334-1552-2. 2009. Disponível em 〈http://bvsms.saude.gov.br/bvs/publicacoes/sus az garantindo saude municipios 3ed p1.pdf $>$ Acesso em 19 de Maio de 2016.

MINISTÉRIO DA SAÚDE. Secretaria de Atenção à Saúde. Departamento de Ações Programáticas Estratégicas (Brasília,DF). Diretrizes de Atenção à Reabilitação da Pessoa com Transtorno do Espectro Autista (TEA). ISBN 978-85-334-2089-2. 2013. Disponível em < http://bvsms.saude.gov.br/bvs/publicacoes/diretrizes_atencao_reabilitacao_pessoa_autismo.pdf> Acesso em 30 de Setembro de 2016.

NETRVAL, Danielle Azarias Defense. Proposta de modelo de indicadores de qualidade para o atendimento oferecido aos indivíduos autistas na cidade de São Paulo. 2014. Tese (Doutorado em Comunicação Humana) - Faculdade de Medicina, Universidade de São Paulo, São Paulo, 2014. Disponível em < http://www.teses.usp.br/teses/disponiveis/5/5162/tde-24062014-151643/pt-br.php> Acesso em 27 de Setembro de 2016.

NOVIKOFF, Cristina. Desafios da Práxis Educacional à Promoção Humana na Contemporaneidade, Rio de janeiro, Reproarte, 2010.

SANTOS, Darci Neves; CARVALHO, Marjorie Moreira de; PINHO, Márcia Andrade; NUNES, Ana Paola Robatto. Atendimento em psiquiatria da infância e adolescência em serviços públicos de Salvador. Rev. Baiana de Saúde Pública. Salvador, v. 29, n. 1, p. 35-42, Jan/Jun. 2005. Disponível em <http://inseer.ibict.br/rbsp/index.php/rbsp/article/viewFile/110/pdf 530> Acesso em 28 de Abril de 2016.

SILVA, Andréa Neiva da; SENNA, Marcos Antônio Albuquerque de. Fundamentos em saúde bucal coletiva. Rio de Janeiro: MedBook, 2013.

SMEHA, Luciane Najar; CEZAR, Pâmela Kurtz. A vivência da maternidade de mães de crianças com autismo. Psicol. estud., Maringá, v. 16, n. 1, p. 43-50, Mar.2011. Disponível em

$<$ http://www.scielo.br/scielo.php?script=sci_arttex\&pid=S1413-

$73722011000100006 \& \operatorname{lng}=\mathrm{en} \& n r m=$ iso >. Acesso em 28 de Abril de 2016. 
UNTOIGLICH, Gisela. As oportunidades clínicas com crianças com sinais de autismo e seus pais. Estilos clin., São Paulo , v. 18, n. 3, p. 543-558, dez. 2013 . Disponível em <http://pepsic.bvsalud.org/scielo.php?script=sci_arttext\&pid=S1415-

$71282013000300008 \& \operatorname{lng}=$ pt\&nrm=iso>. Acesso em 28 de Abril de 2016.

ZAMPIROLI, Wheber Christiano; SOUZA, Valdilene Magno Pinto de. Autismo infantil: uma breve discussão sobre a clínica e o tratamento. Rev. Pediatria Moderna. v. 48, n. 4, p. 126-130. Abr. 2012. Disponível em

<http://www.moreirajr.com.br/revistas.asp?id materia=4975\&fase=imprime $>$ Acesso em 28 de Abril de 2016.

Recebido em: 10/10/2016

Aceito em: 09/01/2017

Endereço para correspondência:

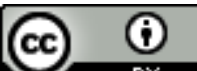

Nome Mayara Andrine do Nascimento

e-mail: mayaraan@gmail.com

Este obra está licenciada com uma Licença Creative Commons Atribuição 4.0 Internacional. 